\title{
Urinary drug screening
}

\section{Dimitri Gerostamoulos \\ Chief toxicologist and \\ manager \\ Victorian Institute of \\ Forensic Medicine \\ Adjunct senior lecturer \\ Department of Forensic \\ Medicine \\ Monash University \\ Melbourne}

\section{Key words}

drug abuse, opioids, saliva

\section{SUMMARY}

Urinary drug screening can detect substances including over-the-counter and prescription drugs. The focus of screening is often illicit drugs.

Simple point-of-care tests, largely immunoassays, can rapidly detect a class of drugs or specific drugs in urine. More complex large scale laboratory screens and confirmatory tests can also be used.

Urine tests can often provide evidence of previous drug consumption. Screening is therefore useful in monitoring abstinence from drug use, the use of drugs in the workplace and in legal disputes.

\section{Introduction}

Drugs, chemicals and other substances consumed by humans are often excreted in the urine, where they may be detected with screening tests. The detection of these compounds is limited only by the assay and cost.

In hospital or pathology laboratories the emphasis is typically on drugs of abuse, screening either for compliance to (e.g. methadone) or abstinence from (e.g. cannabis) particular substances. Hospital screening can also give the clinician additional help in the differential diagnosis and treatment of a patient, but the availability of toxicological analyses varies between hospitals.

Most commonly used drug screening tests involve immunoassay techniques.' These range from so-called bedside or point-of-care testing to more sophisticated laboratory-based immunological tests. Immunoassay can provide fast and reliable results, however the results must be interpreted with caution.

\section{What can be tested?}

The most common drugs tested in urine include amphetamines, benzodiazepines, cannabis, cocaine and opioids. Other tests can screen for more specific compounds, rather than drug classes, such as alcohol, methadone, buprenorphine, phencyclidine and other stimulants (for example cathinones) and designer drugs. Alcohol metabolites such as ethylglucuronide can now be detected in urine and, importantly, for much longer periods than alcohol itself (up to several days). Ethylglucuronide screening has been used clinically as evidence of abstinence from alcohol for patients awaiting liver transplant. ${ }^{2}$

Urinary drug screening can be quite complex with detection of a comprehensive list of targeted and unknown substances. For example, forensic laboratories have the capability to detect a wide range of compounds using immunoassays and other chromatographic techniques (that is gas chromatography/mass spectrometry or liquid chromatography/mass spectrometry). The methods used in these laboratories often aim for a wide range of drugs and metabolites to try to detect as many forensically relevant compounds as possible.

Other laboratories, such as sports testing facilities, can screen for drugs (including steroids and biomarkers). Screens for unknown compounds have been made possible by the advent of new technologies. These laboratories are more specialised than the typical hospital or pathology laboratories and are strictly regulated for the detection of these compounds by accrediting bodies such as the National Association of Testing Authorities, Australia ${ }^{3}$ and the World AntiDoping Agency 4 .

\section{Standards}

An Australian/New Zealand Drug testing standard (AS/NZS 4308:2008) provides guidance on the most common classes of drugs to be tested in urine. ${ }^{5}$ The Australian Standard (AS 4308) was the world's first national standard for medicolegal drug testing. It is designed to ensure the standardisation of procedures for specimen collection and the detection of drugs of abuse. These include cannabis metabolites, cocaine metabolites, benzodiazepines, sympathomimetic amines (amphetamines) and opioids. Urine for medicolegal testing should be collected and analysed by an AS/NZS 4308:2008 accredited organisation. Consultation with the laboratory is useful to find out which compounds can be tested as well as for interpretation of negative or positive findings.

\section{Why test urine for drugs?}

Urine screening can provide an indication that someone has consumed drugs at some point before sampling. Most drugs typically have a detection window of up to 48 hours (Table). This may be shorter for some drugs which are eliminated relatively quickly from the body such as alcohol or gamma- 
hydroxybutyrate. Drugs such as diazepam and cannabis can persist in urine for days or even weeks and so can be detected for longer periods.

The frequency of drug consumption will also have an impact on the effectiveness of urine screening. Acute or once-off use, as in the case of drug-facilitated crime where a drug is used to render someone incapacitated, is more difficult to detect and may challenge the sensitivity of urine screening. People who use drugs more regularly will typically have higher concentrations of drugs in their urine leading to easier detection with possibly longer timeframes. In chronic users, drugs of abuse can be detected in urine for approximately one week after last use, and in extreme cases even longer in cocaine (22 days) and cannabis users (up to three months). ${ }^{6}$ Urine testing will not show when a drug was used, or how much. Importantly, a positive drug result cannot infer impairment at the time the urine was collected.

\section{Results}

Urine testing typically involves a screening test followed by a confirmation test. Confirmation is usually performed on the sample taken for screening.

\section{Screening tests}

Most drugs of interest are first detected by simple immunoassays. These are broad screening tests that are quick, often cheap and effective for showing a positive or negative result. However, as with all screening tests there are limitations to the degree of interpretation that can be inferred from the result. The limitations of immunoassay techniques include false positives as well as false negative results.

A false positive is a screening test that fails to be confirmed using other more sensitive and specific techniques such as gas chromatography/mass spectrometry or liquid chromatography/mass spectrometry. This means that the immunoassay has cross-reacted with some other substance in the urine leading to a false positive result for the substance of interest. Other drugs can trigger such false positive results and the laboratory should have a list of compounds which can cross-react with the screening test. For example, ranitidine can produce a false positive result for amphetamines. It is also worth noting that some foodstuffs can also produce positive results such as poppy seeds for opioids.

A false negative result is possible when the screening test is negative but the confirmatory test is positive. This is less common as negative screening tests are not usually confirmed. When a screening test is negative that is usually the end of the investigation. In a workplace, a false negative test can have farreaching ramifications if an incident occurs after screening and a urine sample test then finds drugs which were missed by the initial screening process. On-site or point-of-care devices must therefore be rigorously tested and validated before use in the field. AS/NZS 4308 states that on-site screening devices be evaluated at $25 \%$ above and $30 \%$ below the level considered positive (these are typically referred to as cut-offs). The Standard also specifies that failure of no more than $10 \%$ of on-site devices is permitted.

When using immunoassay techniques, samples can easily be adulterated to provide a false result. Adulterations are common in patients who undergo clinical compliance testing, for example abstinence control in drug users. Adulterations can include water (leading to dilution of urine), bleach and masking agents (such as diuretics) or other substances that interfere with the screening test. Adulterant checks are also part of a laboratory's capability to detect an invalid specimen. The Standard provides guidance on what to do to avoid adulteration and how to test for adulterants (for example temperature and creatinine checks).

\section{Table Detection of drugs in urine}

\begin{tabular}{ll}
\hline Drug or drug class & Detection times in urine \\
\hline Benzodiazepines (e.g. alprazolam, diazepam, temazepam) & $1-7$ days or longer depending on half-life of drug* \\
\hline Cannabinoids & $3-28$ days depending on frequency of use \\
\hline Cocaine & $1-3$ days \\
\hline Methamphetamine/amphetamine & $2-5$ days \\
\hline Methylenedioxymethamphetamine & $2-5$ days \\
\hline Opioids (e.g. morphine, codeine) & $1-2$ days \\
\hline Steroids (e.g. testosterone, stanozolol) & Days to months depending on the half-life of the steroid \\
\hline * may be longer in chronic users & \\
\hline
\end{tabular}




\section{SELF-TEST QUESTIONS}

True or false?

5. Urinary drug screening can identify previous intoxication with illicit drugs.

6. Cannabis may be present in the urine for up to a month following chronic use.

Answers on page 67

\section{Confirmatory tests}

Confirmation tests are usually required for medicolegal purposes when drug testing is used in the workplace or for family custody disputes in which parents are allegedly using drugs at home in the presence of children. An initial urinary screening test must be confirmed for evidence of drug use. Confirmatory testing is more sensitive and specific than screening tests and confirms the drug of interest as opposed to the drug class.

Clinical laboratories have relied on gas chromatography/mass spectrometry for confirmation, however developments in liquid chromatography/ mass spectrometry technologies over the last 10 years have meant a wider range of compounds can be confirmed simultaneously. Laboratories must demonstrate compliance with requirements to either International Organization for Standardization (ISO) 17025 (for chemical/forensic testing) or ISO 15189 for medical/pathology testing and must be accredited by the National Association of Testing Authorities.

\section{Screening tests other than urine}

The evolution of oral-fluid testing (saliva) both from a policy and technology viewpoint has grown rapidly in recent years. This testing has been used primarily to test drivers for illicit drugs (amphetamines and cannabis) and is now being used in the workplace.
Saliva is easier and safer to collect than urine. Unlike urine, not all drugs are easily detectable in oral fluid, either because of the low concentrations or the short time that drugs are present in saliva. The detection of a drug in oral fluid is normally associated with recent use (up to 24 hours) as the drugs can be related to blood or plasma concentrations and therefore physiological effect. This cannot be inferred from urine as the concentration in urine can only be related to previous consumption and not any effect of the drug.

\section{Conclusion}

Urine screening is an effective tool for monitoring abstinence from drug use, assessing the use of drugs in the workplace and for legal disputes. A number of laboratories can provide testing for drugs of abuse and prescription drugs (for example benzodiazepines and some opioids). Point-of-care tests can provide similar information, however knowledge of which drugs and compounds can be detected as well as interpretation of what the test results mean is essential in maximising the information that can be gained from urinary screening. $<$

\section{Conflict of interest: none declared}

\section{REFERENCES}

1. Cassidy N, Herbert JX, Tracey JA. The availability of toxicological analyses for poisoned patients in Ireland. Clin Toxicol 2010;48:373-9.

2. Staufer K, Andresen H, Vettorazzi E, Tobias N, Nashan B, Sterneck $M$. Urinary ethyl glucuronide as a novel screening tool in patients pre- and post-liver transplantation improves detection of alcohol consumption. Hepatology 2011;54:1640-9.

3. National Association of Testing Authorities, Australia. www.nata.asn.au [cited 2013 Jan 14]
4. World Anti-Doping Agency. www.wada-ama.org [cited 2013 Jan 14]

5. Australian/New Zealand Standard: procedures for specimen collection and the detection and quantitation of drugs of abuse in urine. AS/NZS 4308:2008. Sydney: Standards Australia; 2008.

6. Verstraete AG. Detection times of drugs of abuse in blood, urine, and oral fluid. Ther Drug Monit 2004;26:200-5.

\section{RADAR $)$ \\ Rational Assessment of Drugs and Research}

The April issue of NPS RADAR reviews the evidence and place in therapy for:

- pregabalin (Lyrica) - an alternative analgesic adjuvant for refractory neuropathic pain

- $\quad$ sitagliptin with simvastatin (Juvicor) - fixed-dose combination therapy for type 2 diabetes and hypercholesterolaemia

- imiquimod cream (Aldara) - for superficial basal cell carcinoma

Read the full reviews at www.nps.org.au/radar 